Artículo

\title{
Factores de innovación para la competitividad en la Alianza del Pacífico. Una aproximación desde el Foro Económico Mundial
}

\author{
Florina Arredondo Trapero ${ }^{a}$, José Carlos Vázquez Parra ${ }^{\mathrm{b}, *}$ y Jorge de la Garza ${ }^{\mathrm{c}}$ \\ a Profesor Titular, Departamento de Filosofía y Ética, Tecnológico de Monterrey, Campus Monterrey, Monterrey, Nuevo León, México \\ b Profesor de Cátedra, Departamento de Formación Humanística y Ciudadana, Tecnológico de Monterrey, Campus Guadalajara, Zapopan, Jalisco, México \\ c Profesor Asociado, Departamento de Mercadotecnia y Negocios Internacionales, Tecnológico de Monterrey, Campus Monterrey, Monterrey, Nuevo León, México
}

\section{INFORMACIÓN DEL ARTÍCULO}

\section{Historia del artículo:}

Recibido el 21 de diciembre de 2015

Aceptado el 14 de junio de 2016

On-line el 1 de septiembre de 2016

\section{Códigos JEL:}

019

032

054

Palabras clave:

Innovación

Tecnología

Competitividad

Alianza del Pacífico

\begin{abstract}
R E S U M E N
El presente artículo tiene el objetivo de estudiar, mediante un modelo de regresión, cuáles son las variables que influyen de forma significativa en el pilar de la innovación para la competitividad de los países de América Latina. Posteriormente, se hace un análisis de varianza de las variables innovadoras en el grupo de países que forman parte de la Alianza del Pacífico para contrastarlo con el resto de los países de Latinoamérica y encontrar las variables que marcan una diferencia significativa entre ambos. Se concluye que la aplicación de patentes resulta ser un diferenciador de innovación para la competitividad en la Alianza del Pacífico en relación con el resto de Latinoamérica.
\end{abstract}

(C) 2016 Universidad ICESI. Publicado por Elsevier España, S.L.U. Este es un artículo Open Access bajo la licencia CC BY (http://creativecommons.org/licenses/by/4.0/).
JEL classification:

019

032

054

Keywords:

Innovation

Patent

Competitiveness

Pacific Alliance

\section{Innovation factors for competitiveness in the Pacific Alliance. An approach from the World Economic Forum}

\section{A B S T R A C T}

Through a regression model, this article aims to study which are the variables that influence significantly on the innovation pillar for competitiveness in Latin America. Subsequently, an ANOVA of innovation variables is presented. The group of countries that are part of the Pacific Alliance is contrasted with the rest of Latin American countries to find significant differences between them. The study concludes that patent application is a differentiating innovation variable for competitiveness in the Pacific Alliance in relation to the rest of Latin America.

(C) 2016 Universidad ICESI. Published by Elsevier España, S.L.U. This is an open access article under the CC BY license (http://creativecommons.org/licenses/by/4.0/).

\footnotetext{
* Autor para correspondencia: Av. General Ramón Corona 2514, Nuevo México, 45201 Zapopan, Jalisco, México.

Correo electrónico: jcvazquezp@itesm.mx (J.C. Vázquez Parra).
} 


\section{Fatores de inovação para a competitividade na Aliança do Pacífico. Uma abordagem do Fórum Econômico Mundial}

\section{Classificações JEL:}

019

032

054

Palavras-chave:

Inovação

Tecnologia

Competitividade

Aliança do Pacífico

\section{R E S U M O}

Este artigo tem como objetivo estudar, utilizando um modelo de regressão, quais são as variáveis que influenciam significativamente o pilar da inovação para a competitividade dos países da América Latina. Posteriormente, é realizada uma análise de variância de variáveis inovadoras no grupo de países que fazem parte da Aliança do Pacífico para contrastar com outros países da América Latina e encontrar as variáveis que fazem uma diferença significativa entre ambos. Conclui-se que a aplicação de patentes acaba por ser um diferenciador da inovação para a competitividade na Aliança do Pacífico em relação ao resto da América Latina.

(C) 2016 Universidad ICESI. Publicado por Elsevier España, S.L.U. Este é um artigo Open Access sob uma licença CC BY (http://creativecommons.org/licenses/by/4.0/).

\section{Introducción}

Las circunstancias económicas del mundo, la globalización, la proliferación de tratados comerciales y la nueva sociedad del conocimiento, en apariencia, han puesto de moda a la innovación. Sin embargo, realmente esta no es una temática reciente, ya que desde tiempo atrás se pueden encontrar referentes teóricos que aluden a la innovación como pilar del desarrollo (Bustos, 2015; Ferrari, 2012; Monzón, 2010).

En lo que se refiere a Latinoamérica, se afirma que el papel de algunas de sus economías dependerá de la efectividad que estos países tengan para generar innovación. El éxito de sus economías en lograr una integración satisfactoria a la economía mundial dependerá del grado en que consigan vincular su sistema de investigación con la producción industrial (Castaños, 1994). Tal como lo refiere Peña-Vinces (2008), actualmente se está ante una nueva forma de competir caracterizada por la hipercompetencia global, en donde tienen relevancia tanto los mercados locales como los internacionales. Bajo este nuevo escenario, los modelos tradicionales de competitividad son superados por nuevos modelos, como el del doble diamante (Moon, Rugman y Verbeke, 1995), que considera la competitividad en un escenario internacional y no solo local, donde los países buscan abrir sus fronteras para participar en la economía mundial y a la par deben defender sus mercados locales. Lo anterior crea un nuevo paradigma de competitividad. Se trata de un concepto de competitividad sistémica en donde se vincula a la empresa, la industria, el gobierno y el país. Ante esta nueva situación, la competencia en el mercado implica cooperación y se concreta mediante alianzas estratégicas que surgen entre los diversos actores: competidores, clientes y proveedores, gobiernos y universidades. Es precisamente bajo este paradigma de competitividad sistémica en el escenario internacional que surgen acuerdos regionales, como es el caso de la Alianza del Pacífico. Esta Alianza, que abarca Chile, Colombia, México y Perú, busca entre sus objetivos «construir, de manera participativa y consensuada, un área de integración profunda para avanzar hacia el progreso» (Alianza del Pacífico, 2016, p. 1).

Tomando como referencia lo anterior, el presente artículo tiene el objetivo de estudiar, mediante un modelo de regresión, cuáles son las variables que influyen de forma significativa en el pilar de la innovación para la competitividad del World Economic Forum (WEF) en los países de América Latina. Además, se realiza un análisis de varianza de las variables innovadoras en el grupo de países que forma parte de la Alianza del Pacífico para contrastarlo con el resto de los países latinoamericanos, encontrando las que marcan una diferencia significativa entre ambos grupos. Se concluye que en Latinoamérica aún se tiene una falta de vinculación del sector empresarial para impulsar la innovación. Adicionalmente, la necesidad de una mayor colaboración con la universidad para impulsar la investigación y el desarrollo es una debilidad en la región. Específicamente se encontró que el desarrollo de patentes no forma parte aún de las ventajas competitivas en Latinoamérica. Finalmente, con base en el análisis de varianza entre los países de la Alianza del Pacífico y el resto de Latinoamérica, se concluye que los primeros presentan una ventaja competitiva en innovación por su capacidad de desarrollar patentes, aspecto que puede resultar una diferencia significativa, considerando la precariedad de este factor en el resto de la región. De esta forma, a través de los proyectos desplegados por la Alianza del Pacífico será posible potencializar la transferencia de conocimiento y el desarrollo de tecnología entre los países miembros y avanzar hacia una economía regional basada en el conocimiento.

En la estructura del artículo, como parte del marco teórico, primero se aborda el concepto de competitividad y de innovación, después de presenta el vínculo entre ambos conceptos de acuerdo con el WEF (2014). En una segunda parte se aborda la metodología de investigación empírica, dividida en el modelo de regresión y el análisis de varianza. Finalmente se analizan los resultados y se emiten las conclusiones.

Previo al desarrollo teórico del tema se presenta la siguiente acotación sobre la que se basa el presente estudio. Por una parte, Castro-González, Peña Vinces y Guillen (2016) señalan que los informes del WEF carecen de un análisis estadístico robusto, pues asignan de manera arbitraria el valor que llegan a tener los indicadores de competitividad afectando la confiabilidad de los resultados. Por otra parte, el WEF sin duda alguna ofrece uno de los índices más influyentes con el que es analizada internacionalmente la competitividad en los países (Villarreal y Villarreal, 2002). Con base en lo anterior, en primera instancia se aborda la perspectiva del WEF. Esta perspectiva será complementada con las aportaciones de otros académicos u organismos internacionales como el International Institute for Management Development (IMD) del World Competitiveness Center.

\section{La innovación científica tecnológica para la competitividad}

Acerca de la innovación y su vinculación con la competitividad en las últimas décadas, los países han puesto un gran interés por lograr ventajas competitivas que les permitan alcanzar un crecimiento económico sustentable. Con base en el Comité Intersectorial para la Innovación (CII), una de las formas a que se recurre con más frecuencia para lograr estas ventajas es el desarrollo de una mejor capacidad de innovar, es decir, de "generar nuevos productos, diseños, procesos, servicios, métodos u organizaciones o 
incrementar valor a los existentes» (CII, 2011, p. 5). Por ello es que, según Charreau (2001), la innovación funge como un catalizador de la prosperidad nacional, lo que justifica la inversión en investigación. Sin embargo, también señala que el desarrollo debe redefinirse en términos de la calidad de las ideas generadas y seleccionadas, así como del espectro y la escala de la eventual aplicación en la industria.

Montoya (2004), de manera adicional, argumenta que la innovación científica y tecnológica es el instrumento que puede hacer que el crecimiento y el desarrollo económico de los países subdesarrollados pueda acelerarse o incluso sacarlos del subdesarrollo. Aunque también considera que la innovación tecnológica puede convertirse en un claro factor de desigualdad que afecte aún más a los países que no pueden hacerse de procesos innovadores. Es evidente que el progreso innovador ha ejercido una influencia en el desarrollo de las fuerzas productivas, pero ciertamente no es la única causa del crecimiento económico.

Por consiguiente, se considera que un país con mayores fortalezas en el ámbito de la innovación tendrá mayor capacidad para incrementar su productividad. Lo anterior no solo es por el efecto directo que genera cualquier innovación, sino sobre todo porque estará mejor preparado para enfrentar las incertidumbres generadas por el actual entorno de competencia global y para adaptarse a las condiciones cambiantes de su entorno (CII, 2011). En relación con la innovación y su influencia en el crecimiento productivo de una economía, se identifican 2 factores que influyen. El primero considera el aumento de la cantidad de insumos en el proceso productivo y el segundo se refiere a nuevas formas de obtener una mayor producción o de mayor valor con los mismos recursos, es decir, procesos de innovación. Algunos estudios han encontrado que son las innovaciones específicas las que causan aumentos en la producción de las empresas de ciertos países (Geroski, 1989).

Desde comienzos del siglo XxI, la competitividad y la innovación están siendo fuertemente vinculadas. Los países altamente competitivos son aquellos con inversiones elevadas en las siguientes áreas: investigación y desarrollo, ciencias básicas y aplicadas, inversión en equipos y formación de recursos humanos. Consecuentemente, ello conduce a la invención, la innovación y, por consiguiente, a disponer de tecnología de punta (Freeman, 1993).

Según el Reporte Global de Competitividad del Foro Económico Mundial (WEF, 2014), la innovación tecnológica se constituye como uno de los 12 pilares determinantes para la competitividad de los países. Aunque la mejora de una nación puede gestarse a partir de un aumento en la economía por medio de un incremento en la producción, la construcción de infraestructura, la reducción de la inestabilidad macroeconómica o la mejora del capital humano, a largo plazo la competitividad de un país puede mejorarse, en gran medida, a partir de la inversión en innovación tecnológica.

El Foro Económico Mundial considera importante que la innovación tecnológica, aunque puede llegar a reportar buenos resultados en la competitividad de manera separada, considere el resto de los pilares, pues estos tienden a reforzarse mutuamente. La debilidad en una de las áreas con frecuencia tiene un impacto negativo en otra. Por ejemplo, la fuerza laboral saludable y bien educada que contemplan los pilares 4 y 5 resultan determinantes para generar innovación, la cual también puede verse afectada si no hay financiamiento suficiente (pilar 8) o un mercado de productos eficiente (WEF, 2014).

De esta manera es que se puede identificar a la innovación como una piedra angular dentro de la competitividad de las empresas y de las naciones, y por ello es necesario invertir en el diseño y desarrollo de productos y procesos de vanguardia. Este reto solo llega a conseguirse dentro de ambientes propicios para la actividad innovadora, a partir del apoyo de los sectores tanto públicos como privados (Villarreal y Villarreal, 2002). Por lo tanto, es necesario que los países que forman parte de la Alianza del Pacífico consideren a la innovación como un elemento determinante para la mejora de sus ventajas competitivas, sin olvidar el resto de factores que pueden marcar una clara diferencia en sus resultados.

Según el Reporte Global de Competitividad (WEF, 2014), la innovación no puede generar los beneficios esperados sin que los países apuesten a una inversión suficiente en áreas de investigación y desarrollo a partir de la presencia de instituciones de investigación científica de alta calidad. Además, es necesario buscar la colaboración entre las universidades y la industria, así como la protección de la propiedad intelectual y el acceso a financiamiento (Peña-Vinces y Urbano, 2014). En esta misma línea, Arredondo, de La Garza y Villa (2015) argumentan que la colaboración fomenta la confianza, ayuda a que la organización innove y logre generar ventajas competitivas. Solo a partir de un equilibrio entre estos factores es posible abrir una senda óptima a la mejora en la competitividad por medio de procesos de innovación.

De acuerdo con el Centro Internacional de Investigación de Información sobre Economía Pública, Social y Cooperativa (CIRIEC, 2008), las sociedades y sus economías requieren ser innovadoras, pues la innovación es determinante en el diseño de políticas públicas y políticas empresariales. De esta forma, la tendencia es hacia un enfoque de la innovación no solo en su dimensión tecnológica, sino también en las dimensiones organizativas, sociales, institucionales, culturales y políticas. Estas dimensiones no fueron contempladas anteriormente, lo cual influyó en la ineficiencia de numerosas políticas de desarrollo.

En este sentido, la innovación se constituye como una de las inversiones más importantes de las naciones. Además de ser un pilar determinante para mejorar la competitividad, también permite generar nuevo conocimiento y plantear soluciones a problemas relacionados con la salud, el medio ambiente, la pobreza y la seguridad, entre otros. Gracias también a la innovación se puede lograr un crecimiento económico sostenido al generar mejoras en la productividad (Bustos, 2015). En resumen, la innovación eleva la calidad de vida de las personas (Cámara de Diputados y Diseña México, 2008). Por este motivo es necesario establecer políticas a corto, mediano y largo plazo que permitan fortalecer la cadena establecida entre educación, ciencia básica y aplicada, tecnología e innovación (CII, 2011).

Diferentes organismos internacionales han hecho énfasis en el importante papel que juega la innovación en el mejoramiento de la calidad de vida de las personas, especialmente de las que viven en países emergentes o en desarrollo (González y Martín, 2013). Instituciones con este fin son el Instituto de Innovación Social de la Universidad Ramón Llull, la Escuela Superior de Administración y Dirección de Empresas (ESADE, 2015) y el Centro de Innovación Social de la Universidad de Deusto (Deusto, 2015). Estos centros buscan contribuir por medio de la investigación a la formación y la divulgación en temas de responsabilidad social empresarial, liderazgo, emprendimiento social e innovación. Estas instituciones consideran que estos factores llegan a constituirse como ejes centrales en el fomento de una armonía territorial de los países. Además, promueven el que las personas se constituyan en motores de cambio suscitando un modelo de desarrollo sostenible, socialmente responsable, generador de riqueza y respetuoso con el medio ambiente.

El Foro Económico Mundial, en su Reporte Global de Competitividad (WEF, 2014), ha señalado que la innovación es uno de los pilares fundamentales para el desarrollo de ventajas competitivas dentro de los países. No basta con el aumento de producción y la inversión extranjera para mejorar o promover un desarrollo sostenible de un país o región. Es necesario que los países inviertan en innovaciones que promuevan el mejoramiento de la infraestructura rural y urbana. Adicionalmente, se deben combatir los problemas sociales que provocan inestabilidad macroeconómica y apoyar la mejora del capital humano. Solo de esta manera la innovación llega 
a configurarse como una piedra angular en el mejoramiento de la calidad de vida de los habitantes.

En este mismo sentido, el WEF (2014) reconoce que la innovación puede surgir tanto de un nuevo conocimiento tecnológico como no tecnológico. Si bien las innovaciones no tecnológicas pueden ser relevantes, tales como el desarrollo de nuevas habilidades organizacionales, no resultan ser tan estratégicas para el logro de la competitividad internacional de un país.

Como ya se ha dicho, el último pilar de la competitividad que mide el Foro Económico Mundial se centra en la innovación tecnológica. Aunque la competitividad se puede lograr mediante la mejora de las instituciones, la construcción de infraestructura, la reducción de la inestabilidad macroeconómica o el mejoramiento del capital humano, todos estos factores con el tiempo tienden a generar rendimientos decrecientes. Lo mismo aplica para la eficiencia en mano de obra, financiera o de bienes de mercado. Con base en lo anterior se afirma que, a largo plazo, el nivel de vida de un país se puede mejorar sustancialmente si hay innovación tecnológica.

El WEF (2014) reconoce que históricamente la innovación tecnológica ha sido un punto de quiebre para ganar productividad en la economía de diversos países, como actualmente se hace referencia a la revolución digital.

La innovación no solo está transformando la forma en que las cosas se están haciendo, también está abriendo nuevas posibilidades en términos de productos y servicios. Si bien la innovación es un punto estratégico entre las naciones, no tiene la misma influencia en diferentes regiones. La innovación es particularmente importante para las economías cercanas a las fronteras del conocimiento y es menos relevante para las economías que solo integran y adaptan tecnologías exógenas (Naranjo-Valencia y Calderón-Hernández, 2015).

Aunque los países menos avanzados pueden mejorar su productividad mediante la adopción de las tecnologías existentes o haciendo mejoras incrementales, para aquellos que han llegado a la etapa de innovación desarrollada, esto ya no es suficiente para incrementar su productividad (García-Osorio, Quintero-Quintero y Arias-Pérez, 2014). Las empresas de estos países deben diseñar y desarrollar productos de vanguardia y procesos para mantener su ventaja competitiva y avanzar hacia actividades de mayor valor añadido. Tal progreso se logrará con un entorno que conduzca a la actividad innovadora y con el apoyo del sector público y privado (De Arteche, Santucci y Welsh, 2013).

Para poder lograr una mayor innovación se precisa una mayor inversión en investigación y desarrollo (I+D), especialmente del sector privado. También se requiere la presencia de una alta calidad científica en las instituciones de investigación que puedan generar los conocimientos básicos para desarrollar nuevas tecnologías. Adicionalmente, se demanda una amplia colaboración en la investigación y el desarrollo tecnológico entre las universidades y la industria, así como la protección de la propiedad intelectual (Castro-González et al., 2016; Peña-Vinces y Urbano, 2014).

Finalmente, el WEF (2014) advierte que, a la luz de la lenta recuperación económica y el aumento de las presiones fiscales que enfrentan las economías avanzadas, es importante que los sectores públicos y privados resistan a la presión de reducir la inversión en $\mathrm{I}+\mathrm{D}$, pues es muy importante para el crecimiento sostenido en el futuro.

\subsection{La innovación en los países miembros de la Alianza del Pacífico}

Para tener una mejor perspectiva de lo señalado por el Fondo Económico Mundial, se presenta la tabla 1, la cual refleja la situación en Latinoamérica del pilar 12, innovación del Reporte Global de Competitividad.
A continuación se comparten los puntos de análisis más relevantes de la innovación para la competitividad de los países que comprenden la Alianza del Pacífico.

Chile es el país que el WEF (2014) considera como la economía más competitiva de Latinoamérica. Gran parte de sus ventajas radican en contar con una fuerte estructura institucional, con un bajo nivel de corrupción y un gobierno eficiente. Sin embargo, Chile necesita hacer grandes esfuerzos para enfrentarse a algunas debilidades que pueden afectar a su desarrollo, como lo es la poca diversidad de su economía, una reducida inversión en innovación, una mejora en la calidad educativa y una mano de obra con poca capacidad para el desarrollo de proyectos innovadores (Amorós, Felzensztein y Etchebarne, 2012). Este tipo de factores han dado lugar a una transición lenta de una economía basada en la producción a una economía basada en el conocimiento, lo cual pudiera ser un detonante para el desarrollo del país. En materia de innovación, Chile precisa capitalizar sus fortalezas innovadoras en la región, en las que destacan: la colaboración universidad-industria en investigación, el apoyo gubernamental en productos de tecnología avanzada, la disposición de científicos para innovar, y continuar avanzando en la producción de patentes, que es la más elevada en la región. Adicionalmente, Chile precisa impulsar su capacidad de innovación, la calidad de las instituciones de investigación científicas, así como la inversión privada en investigación y desarrollo (Amorós, Basco y Romaní, 2014). De esta forma podrá potencializar su capacidad innovadora, no solo en Latinoamérica, sino en el entorno global.

Colombia es un país que el WEF (2014) considera que ha tenido un crecimiento económico constante, aunque la mayoría de sus recursos provienen de la producción petrolera, lo que hace que su industria sea poco diversificada. Además, se señala la necesidad de mejorar la calidad de su sistema educativo, pues esto no consigue responder a los requerimientos productivos que precisan las empresas innovadoras y sofisticadas. Esta situación, aunada a una baja inversión privada en investigación y desarrollo y una limitada calidad de la investigación en instituciones científicas, arroja una tasa de innovación con un crecimiento poco significativo. Por otra parte, dentro de la región se destaca por el apoyo gubernamental para desarrollo de productos con tecnología avanzada como parte de su potencial innovador.

Por otra parte, México se ve afectado por sus políticas públicas y económicas. Con base en el WEF (2014), México debe fortalecer sus instituciones, sobre todo en temas como la corrupción y la inseguridad. México debe impulsar su capacidad de innovación por medio de actividades económicas de valor agregado, así como fomentar el uso de tecnologías de la información, pues esto reduce su capacidad de desarrollar ventajas competitivas. En materia de innovación, para México es necesario sacar ventaja de las fortalezas innovadoras en las que destaca regionalmente: la capacidad de innovación, la inversión privada en investigación y desarrollo, la colaboración universidad-industria en investigación y desarrollo y la disponibilidad de investigadores. Adicionalmente, México tendrá que poner especial atención en estas áreas de oportunidad que aún refleja como país: el apoyo gubernamental en productos de tecnología avanzada, incrementar la generación de patentes, así como la calidad de las instituciones de investigación científica para poder impulsar la innovación como pilar de competitividad en Latinoamérica.

De acuerdo con el análisis del WEF (2014), la situación de Perú impone retos importantes. En el país andino el deficiente nivel educativo ha generado una brecha muy profunda entre los requerimientos de las empresas y la mano de obra que se ofrece, lo que da lugar a un limitado sistema de investigación, una casi nula capacidad de diversificación de su economía y, por ende, una baja tasa de innovación. Perú también requiere impulsar su capacidad de innovación, la calidad de las instituciones de investigación científica, 


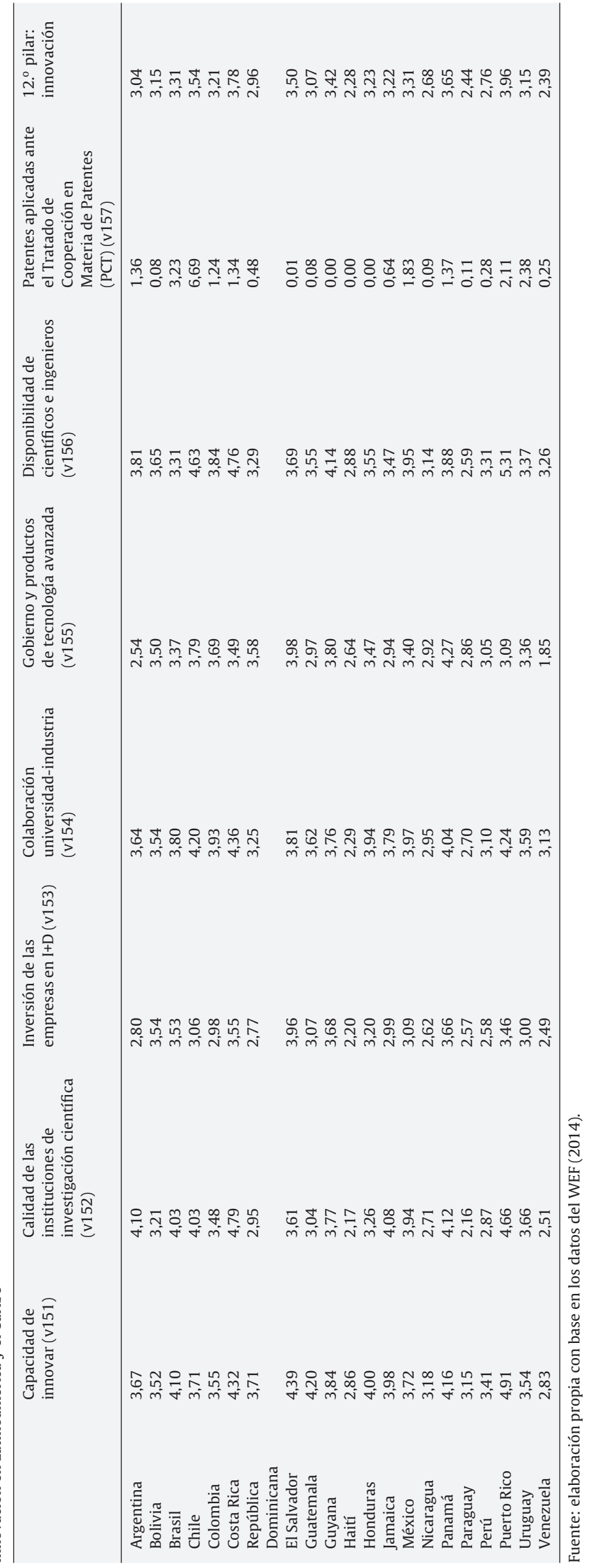


la inversión privada en investigación y desarrollo, la colaboración universidad-industria en investigación y desarrollo, el apoyo gubernamental en productos de tecnología avanzada, así como la disposición de científicos e ingenieros para poder impulsar la innovación como ventaja competitiva en la región.

En general, estas consideraciones del WEF (2014) tienen una visión más clara del panorama de innovación en los países miembros de la Alianza del Pacífico, y aunque existen factores que resultan determinantes y que influyen en el lento nivel de crecimiento de la región, según el informe del IMD, esta alianza ha resultado muy benéfica para el desarrollo de la competitividad de estas naciones.

Con base en las observaciones de León y Ramírez (2014), la Alianza del Pacífico debe ser vista como un proyecto de integración económica y comercial en Latinoamérica, mismo que parece haber trazado una división vertical de la región. Con este criterio, los autores perfilan dos Américas Latinas: la del Atlántico, con una política más paternalista, con una instrumentación restrictiva y desconfiada de la globalización, y la del Pacífico, que opta por el libre mercado y comercio. Esto tiene claras consecuencias en los países miembros, ya que a pesar de una degradación continua debida a las decisiones en materia de políticas económicas adoptadas durante los últimos 30 años, el potencial económico de Chile, Colombia, México y Perú sigue siendo importante.

Según el Ranking 2015 del IMD World Competitiveness Center, Chile sigue siendo el país más competitivo de Latinoamérica, aunque cayó del puesto 31 en el 2014 al 35, seguido por México (lugar 39), Colombia (lugar 51) y Perú (lugar 54), en una escala de 61 países. Según este Instituto, uno de los factores fundamentales para el desarrollo de la competitividad en los países es el ambiente económico, el cual debe buscar ser lo más estable y predecible posible. Algunos Estados latinoamericanos como Argentina, Brasil y Venezuela se ven muy afectados en este aspecto, pues tienen un alto nivel inflacionario, una política económica proteccionista y altas tasas arancelarias para la importación, lo que no incentiva la ganancia en las empresas y mengua su interés por competir. Brasil específicamente ha perdido varios escaños en los últimos años, y esto se ha debido en gran medida a la necesidad de impulsar la educación de sus habitantes, a sus altos niveles de desigualdad y a una baja proporción de comercio frente al PIB. Estas políticas básicamente debilitan a la iniciativa privada y afectan directamente la competitividad y la innovación (IMD, 2015). Sin embargo, según estudios de Castro-González et al. (2016) la competitividad brasileña podría ser más sobresaliente si se hiciera un estudio longitudinal, lo que ocasionaría que la competitividad chilena pasara a un tercer sitio después de Argentina (2) y Brasil (1). En este sentido se reconoce que hay una limitación en el presente estudio, ya que el artículo está basado únicamente en la base de datos del WEF, por lo que no está de más aclarar que hay otras aproximaciones al tema que pueden aportar una perspectiva diferente.

El Informe del IMD señala que la intensa relación entre México y la economía norteamericana mejora considerablemente su situación de comercio exterior, pues es el único país de la Alianza del Pacífico que ha tenido un crecimiento y un manejo de riesgos estable. Sin embargo, esto no es suficiente, ya que México debe buscar la manera de fortalecer sus instituciones así como mejorar su precaria situación social, ya que ambos puntos terminan generando una gran incertidumbre en los nuevos inversionistas extranjeros. Por otro lado se tiene a Perú, quien ha llamado mucho la atención en cuanto al control de sus finanzas públicas, las cuales habían sido un punto alarmante desde el año 2009. A pesar de una relativa estabilidad, esta nación andina sigue teniendo grandes debilidades, como lo son su carente cooperación de tecnología y el bajo nivel educativo de su mano de obra.

Según el informe del IMD, la Alianza del Pacífico ha beneficiado bastante a sus miembros; sin embargo, aún es necesario trabajar en el mejoramiento de algunos elementos de sus economías nacionales, como por ejemplo la generación de un capital humano desarrollado, el fortalecimiento de la innovación y el intercambio de tecnología, elementos que resultan fundamentales para la competitividad de las naciones (IMD, 2015). Además, es necesario que los países de la Alianza del Pacífico mejoren sus prácticas públicas, optando por políticas económicas que relacionen la demanda externa de producción industrial como medio para promover la innovación, y no a partir de políticas de promoción del comercio exterior desvinculadas de la producción y el desarrollo tecnológico (León y Ramírez, 2014).

Como se pudo apreciar en el informe del WEF, existen factores que resultan determinantes y que influyen en el lento nivel de crecimiento en la región, ya que uno de los mayores problemas de Latinoamérica reside en gran medida en la falta de inversión en el desarrollo de los proyectos de innovación existentes (WEF, 2014). Lamentablemente, la innovación no es suficiente para mejorar la situación de un país, ya que si los intentos por generar proyectos innovadores no cuentan con un apoyo adecuado para su implementación, a causa de tardías reformas empresariales, insuficiencia en la asignación de recursos, falta de calidad educativa o una carente presencia de políticas públicas que promuevan su ejecución y desarrollo, difícilmente se puede llegar a tener resultados benéficos.

Tanto el informe del IMD como el informe del WEF refieren como pilar a la innovación en este grupo de países que conforma la Alianza del Pacífico. Por lo tanto, para el éxito de la Alianza se precisa fortalecer las instituciones de investigación científica, el decidido apoyo público y privado, la estabilidad económica y los vínculos universidad-industria en materia de investigación y desarrollo.

\section{Metodología}

La sección de metodología se divide en 2 subapartados. En el primero se hace referencia al problema y objetivo de la investigación, y en el segundo se explica el modelo de regresión múltiple generado.

\subsection{Problema y objetivo de la investigación}

Tal como se abordó en el marco teórico, uno de los pilares más importantes para medir la competitividad en las naciones es la innovación. Sin embargo, en el escenario internacional, Latinoamérica sigue presentando rezagos en este tema. Con este objetivo, el presente artículo trata de identificar cuáles son las variables que influyen significativamente dentro del pilar de la innovación para la competitividad en las economías de Latinoamérica, particularmente considerando los países miembros de la Alianza del Pacífico.

En el caso de este gremio de países, son pocos los estudios que han analizado las variables que son consideradas en la medición de la innovación. El análisis sobre el tema se remite, en la mayoría de los casos, a estrategias de innovación seguidas por empresas o instituciones. Esta carencia de análisis plantea un problema, que es la falta de referentes para dar una mejor orientación acerca de las estrategias para incrementar la innovación como parte de la competitividad de la región.

De esta forma, la presente investigación empírica fue realizada con el fin de responder a esta inquietud, para lo cual se plantean hipótesis de investigación. Tales hipótesis tienen como propósito identificar las variables específicas que inciden sobre en el pilar de innovación para la competitividad de los países en Latinoamérica, e identificar posteriormente en cuáles de ellos hay diferencias significativas entre los países que conforman la Alianza del Pacífico y el resto de la región latinoamericana. 


\subsection{Modelo de regresión múltiple}

El análisis de regresión es el método estadístico que se ha seleccionado para analizar las relaciones entre las variables y su efecto en la innovación para la competitividad en los países latinoamericanos que son estudiados por el WEF (2014). Mediante un modelo de regresión lineal múltiple se trata de explicar el comportamiento de la variable endógena o dependiente, que es la innovación para la competitividad de los países de Latinoamérica, en función de un conjunto de variables explicativas que conforman el pilar 12 de innovación del WEF (2014). El objetivo de este análisis es la obtención de un modelo que ayude a comprender la influencia de este conjunto de variables en la innovación de la región.

El diseño de la investigación es no experimental transeccional, pues se careció del control de cualquier variable y los datos empleados fueron recuperados del sitio del WEF (2014). El estudio se realizó en un colectivo de 21 países latinoamericanos que fueron evaluados por el WEF (2014) en el área de innovación.

Las variables (v) que fueron estudiadas para medir el modelo de regresión múltiple se señalan a continuación:

- Capacidad para innovar (v151). Está basada en el grado en que las compañías son percibidas para adquirir tecnología, conducir investigación formal y ser pioneras en sus propios productos y procesos. Hace referencia a la medida en que las empresas pueden generar ideas que resulten en nuevos productos, servicios o modelos de negocios. La capacidad de innovar permite que las instituciones se encuentren en mejora continua y en constante renovación.

- Calidad de las instituciones de investigación científica (v152). Se relaciona directamente con la generación de centros de investigación y la percepción que se tiene de la calidad de los mismos en comparación a sus análogos en otros países. Se considera instituciones de investigación científica a las organizaciones que tienen dentro de sus objetivos la generación de conocimiento científico y su promoción en la solución de problemas. Además se toma en cuenta si forman recursos humanos de alta especialización, fomentan la vinculación entre la academia y los sectores público, privado y social, promueven la innovación científica, tecnológica y social, fomentan la difusión y la divulgación de la ciencia y la tecnología, así como si promueven la cultura científica y tecnológica.

- Inversión de las empresas en I+D (v153). Hace referencia a la cantidad de recursos económicos o humanos que invierten las empresas en la investigación y el desarrollo, aspectos que se encuentran comúnmente asociados a la innovación. La inversión en I+D puede darse en 3 clases: investigación básica, investigación aplicada a procesos y el desarrollo tecnológico (Rivera Godoy, 2015). La promoción de la inversión en I+D casi siempre tiene como resultado un mejoramiento en la capacidad de innovación, aunque para ello es necesario no solo el factor económico, sino también contar con recursos humanos de alto nivel (García, Barona y Madrid, 2013).

- Colaboración universidad-industria I+D (v154). Es la colaboración que existe entre las personas al momento de compartir ideas de innovación entre las universidades o centros de investigación y las empresas. Las instituciones académicas deben establecer políticas de vinculación y transferencia tecnológica que incentiven y promuevan la atracción de beneficios económicos a las empresas con las que se vinculan a través del licenciamiento de patentes o de tecnologías no patentables. A través de este tipo de vinculaciones se evita que la industria tienda a comprar tecnología en vez de recurrir a la colaboración con las instituciones académicas de investigación científica, lo que afecta plenamente la promoción de la innovación. Existe una gran necesidad de promover la investigación y el desarrollo colaborativo, facilitando así la relación entre ambos sectores y generando un fortalecimiento en la investigación y desarrollo tecnológico a nivel académico (Sanchez, García y Mendoza, 2015).

- Gobierno y productos de tecnología avanzada (v155). Hace referencia al proceso de decisión que antecede a las compras tecnológicas que hacen los gobiernos. Para poder señalar que un Estado adquiere prudentemente productos tecnológicos avanzados, se espera que su decisión se encuentre sustentada en técnicas de rendimiento y en su capacidad de valorar la innovación del producto y no simplemente en el precio de los mismos. Los productos tecnológicos que adquiere el Estado tienden a ser fundamentales en el ámbito de la infraestructura y los servicios, así que la calidad de los mismos se relaciona directamente con la mejora en la vida de sus ciudadanos. Este apartado también tiene relación con la cantidad de productos tecnológicos que el Estado adquiere de entes externos, lo cual es paralelo a su capacidad de innovación en este ámbito.

- Disponibilidad de científicos e ingenieros (v156). Considera la cantidad de investigadores en I+D con los que cuenta la nación, es decir, los profesionales involucrados en la concepción o creación de nuevos conocimientos, productos, procesos, métodos o sistemas, así como en la gestión de los proyectos en cuestión. Los Estados deben disponer de recursos humanos de alto nivel si su pretensión es promover la mejora competitiva en el rubro científico tecnológico, ya que la inversión económica resulta insuficiente si no se cuenta con individuos capacitados para generar nuevos proyectos de innovación.

- Patentes aplicadas ante el Tratado de cooperación en materias de patentes (v157). La globalización ya no es algo exclusivo de las empresas multinacionales, por lo que cualquier persona que cuente con un producto o proceso comercialmente atractivo debe considerar su proyección en mercados internacionales y, con ello, la protección de sus derechos como innovador. La aplicación de patentes es el número de aplicaciones presentadas ante el Tratado de cooperación de patentes (PCT) por cada millón de habitantes. El cálculo se obtiene tomando en cuenta la prioridad por fecha y la nacionalidad del inventor.

El modelo a generar trata de predecir el comportamiento del pilar de innovación en Latinoamérica (v150) en función de las anteriores variables. Se plantea un modelo de regresión lineal múltiple que contempla las 7 variables listadas y una variable dependiente que es el pilar de innovación en Latinoamérica. Al plantear el modelo se espera que un aumento en las variables independientes influya en un aumento en la variable dependiente. Se espera, por tanto, que los índices de las variables independientes sean positivos.

- H1: la capacidad para innovar (v151) influye positivamente sobre el pilar de la innovación para la competitividad en Latinoamérica.

- H2: la calidad de las instituciones de investigación científica (v152) influye positivamente sobre el pilar de la innovación para la competitividad en Latinoamérica.

- H3: la inversión de las empresas en I+D (v153) influye positivamente sobre el pilar de la innovación para la competitividad en Latinoamérica.

- H4: la colaboración universidad-industria I+D (v154) influye positivamente sobre el pilar de la innovación para la competitividad en Latinoamérica.

- H5: el gobierno y productos de tecnología avanzada (v155) influyen positivamente sobre el pilar de la innovación para la competitividad en Latinoamérica.

- H6: la disponibilidad de científicos e ingenieros (v156) influye positivamente sobre el pilar de la innovación en Latinoamérica. 
Tabla 2

Estadística de regresión

\begin{tabular}{lc}
\hline Coeficiente de correlación múltiple & 0,998 \\
Coeficiente de determinación $\mathrm{R}^{2}$ & 0,996 \\
$\mathrm{R}^{2}$ ajustado & 0,994 \\
Error típico & 0,035036 \\
Número de observaciones & 20 \\
\hline
\end{tabular}

Fuente: elaboración propia con base en datos del WEF (2014).

- H7: las patentes aplicadas (v157) influyen positivamente sobre el pilar de la innovación para la competitividad en Latinoamérica.

Dado lo anterior, la ecuación de la regresión es la siguiente:

$$
\begin{aligned}
\mathrm{Y}_{\mathrm{e}}= & \mathrm{B}_{0}+\mathrm{B}_{1} \mathrm{X}_{1 \mathrm{i}}+\mathrm{B}_{2} \mathrm{X}_{2 \mathrm{i}}+\mathrm{B}_{3} \mathrm{X}_{3 \mathrm{i}}+\mathrm{B}_{4} \mathrm{X}_{4 \mathrm{i}}+\mathrm{B}_{5} \mathrm{X}_{5 \mathrm{i}}+\mathrm{B}_{6} \mathrm{X}_{6 \mathrm{i}} \\
& +\mathrm{B}_{7} \mathrm{X}_{7 i}+\mathrm{e}_{\mathrm{i}}
\end{aligned}
$$

Donde los parámetros B de la anterior ecuación miden la intensidad media de los efectos de las variables explicativas (v151 ...v157) sobre la variable a explicar (v150) y se obtienen al tomar las derivadas parciales de la variable a explicar respecto a cada una de las variables explicativas. Por otra parte, $Y_{\mathrm{e}}$ se refiere al valor de predicción del pilar de innovación del WEF, estimado por el modelo; $\mathrm{B}_{0}$ se refiere a los parámetros desconocidos; mientras que $X_{1 \mathrm{i}} \ldots \mathrm{X}_{7 \mathrm{i}}$ son las variables independientes (v151, v152, v153, v154, v155, v156, v157) que constituyen el pilar de innovación del WEF; finalmente, $\mathrm{e}_{\mathrm{i}}$ son los términos de residuos o errores.

\section{Resultados del modelo}

De acuerdo con la regresión, los resultados del modelo se muestran en la tabla 2.

El análisis de regresión lineal múltiple realizado demuestra que el 0,994 (tabla 2) de la variable pilar de innovación está determinado por las variables v151, v152, v153, v154, v155, v156. Además demuestra que existe un efecto de regresión altamente significativo de las variables referidas, dado que se obtuvo para las 6 variables un $\mathrm{p}<0,000$ (tabla 3), el cual es menor que el nivel crítico de comparación (alpha $\leq 0,05$ ).

En la tabla 3 se puede observar que el modelo de mejor ajuste resulta altamente significativo.

Este modelo de regresión lineal múltiple permite predecir que, partiendo del intercepto $-0,006$, por cada aumento en las variables v151 (0,231), v152 (0,264), v153 (0,089), v154 (0,127), v155 $(0,218)$ y v156 $(0,240)$ se influye en la variable dependiente en esa magnitud.

A partir de la aplicación del modelo se presenta la siguiente ecuación, la cual recoge los resultados de la tabla 4 .

$$
\begin{aligned}
\mathrm{Y}_{\mathrm{e}}= & -0,006+(0,231) V 151+(0,264) V 152+(0,089) V 153 \\
& +(0,127) V 154+(0,218) V 155+(0,240) V 156+\text { ei }
\end{aligned}
$$

Como se puede observar en los coeficientes estandarizados (beta) (tabla 4), las variables capacidad para innovar (v151), calidad de las instituciones de investigación científica (v152), gobierno y productos de tecnología avanzada (v155), disponibilidad de científicos e ingenieros (v156) tienen una doble influencia en el pilar de la innovación en relación con las otras 2 variables: inversión de las empresas en I+D (v153) y colaboración universidad-industria I+D (v154), cuya influencia, aunque significativa, es menor.

Es interesante analizar cómo para Latinoamérica la falta de vinculación del sector empresarial para impulsar la innovación, así como la necesidad de una mayor colaboración con la universidad para impulsar la investigación y el desarrollo, es una debilidad en la región. Lo anterior termina afectando la competitividad de este gremio de países.

Finalmente, como pudiera esperarse en el pilar de innovación del WEF, la mayoría de las variables influyen de forma significativa

Tabla 3

Modelo de regresión

\begin{tabular}{llcc}
\hline Modelo & Suma de cuadrados & Grados de libertad & Media cuadrática \\
\hline 6 Regresión & 3,958 & 6 & 0,660 \\
$\quad$ Residuo & 0,017 & 14 & 0,001 \\
$\quad$ Total & 3,975 & 20 & 537,317 \\
\hline
\end{tabular}

Modelo 6. Predictores: (constante), capacidad para innovar (v151), calidad de las instituciones de investigación científica (v152), inversión de las empresas en I+D (v153), colaboración universidad-industria I+D (v154), gobierno y productos de tecnología avanzada (v155), disponibilidad de científicos e ingenieros (v156).

\begin{tabular}{|c|c|c|c|c|c|c|c|}
\hline \multirow[t]{2}{*}{ Modelo } & \multicolumn{2}{|c|}{$\begin{array}{l}\text { Coeficientes no } \\
\text { estandarizados }\end{array}$} & \multirow{2}{*}{$\begin{array}{l}\text { Coeficientes } \\
\text { estandarizados. } \\
\text { Beta }\end{array}$} & \multirow[t]{2}{*}{$\mathrm{T}$} & \multirow[t]{2}{*}{ Sig. } & \multicolumn{2}{|c|}{$\begin{array}{l}\text { 95\% intervalo de confianza } \\
\text { para B }\end{array}$} \\
\hline & B & Error estándar & & & & Límite inferior & Límite superior \\
\hline \multicolumn{8}{|l|}{6} \\
\hline Constante & $-0,006$ & 0,070 & & $-0,085$ & 0,934 & $-0,157$ & 0,145 \\
\hline Capacidad para innovar (v151) & 0,200 & 0,030 & 0,231 & 6,686 & 0,000 & 0,136 & 0,265 \\
\hline $\begin{array}{l}\text { Calidad de las instituciones de } \\
\text { investigación científica (v152) }\end{array}$ & 0,157 & 0,027 & 0,264 & 5,855 & 0,000 & 0,100 & 0,215 \\
\hline $\begin{array}{l}\text { Inversión de las empresas en I+D } \\
\text { (v153) }\end{array}$ & 0,086 & 0,035 & 0,089 & 2,437 & 0,029 & 0,010 & 0,161 \\
\hline $\begin{array}{l}\text { Colaboración } \\
\text { universidad-industria I+D (v154) }\end{array}$ & 0,107 & 0,042 & 0,127 & 2,561 & 0,023 & 0,017 & 0,196 \\
\hline $\begin{array}{l}\text { Gobierno y productos de } \\
\text { tecnología avanzada (v155) }\end{array}$ & 0,177 & 0,021 & 0,218 & 8,287 & 0,000 & 0,132 & 0,223 \\
\hline $\begin{array}{l}\text { Disponibilidad de científicos } \\
\text { e ingenieros ( } v 156)\end{array}$ & 0,169 & 0,024 & 0,240 & 7,150 & 0,000 & 0,118 & 0,220 \\
\hline
\end{tabular}

Fuente: elaboración propia.

Tabla 4

Coeficientes del modelo de regresión

Variable dependiente: 12 .- pilar: Innovación

Fuente: elaboración propia. 
Tabla 5

Prueba de ANOVA para ambos grupos de países

\begin{tabular}{|c|c|c|c|c|c|}
\hline & Suma de cuadrados & Grados de libertad & Media cuadrática & $\mathrm{F}$ & Sig. \\
\hline \multirow[t]{3}{*}{ PCT aplicación de patentes por millón de habitantes } & Entre grupos & 1 & 9,520 & \multirow[t]{3}{*}{4,455} & \multirow[t]{3}{*}{0,048} \\
\hline & Dentro de grupos & 19 & 2,137 & & \\
\hline & Total & 20 & & & \\
\hline
\end{tabular}

Fuente: elaboración propia.

en el modelo. Por otra parte, solo una variable fue excluida del modelo: patentes aplicadas (v157), ya que su influencia no resultó ser significativa en la innovación de Latinoamérica. La calificación de la aplicación de patentes de la mayoría de los países de Latinoamérica es visible y establemente baja, de ahí su baja influencia en el modelo. En resumen, el desarrollo de patentes aún no forma parte de las ventajas competitivas en Latinoamérica, por lo que se puede identificar como una limitación para pasar a ser una economía regional basada en el conocimiento.

\subsection{Análisis de varianza}

Con el fin de observar la distinción entre las variables que constituyen el pilar de la innovación para la competitividad, separando el grupo de países de la Alianza del Pacífico del resto de los países que conforman Latinoamérica, se llevaron a cabo pruebas de análisis de varianza (ANOVA). Las pruebas de ANOVA permiten determinar si hay una varianza significativa entre las distintas variables del pilar de innovación analizando ambos bloques de países.

- H8: hay diferencia significativa en los países que conforman la Alianza del Pacífico y el resto de los países de Latinoamérica en relación con la capacidad para innovar (v151).

- H9: hay diferencia significativa en los países que conforman la Alianza del Pacífico y el resto de los países de Latinoamérica en relación con la calidad de las instituciones de investigación científica (v152).

- H10: hay diferencia significativa en los países que conforman la Alianza del Pacífico y el resto de los países de Latinoamérica en relación con la inversión de las empresas en $I+D$ (v153).

- H11: hay diferencia significativa en los países que conforman la Alianza del Pacífico y el resto de los países de Latinoamérica en relación con la colaboración universidad-industria $I+D$ (v154).

- H12: hay diferencia significativa en los países que conforman la Alianza del Pacífico y el resto de los países de Latinoamérica en relación con el gobierno y productos de tecnología avanzada (v155).

- H13: hay diferencia significativa en los países que conforman la Alianza del Pacífico y el resto de los países de Latinoamérica en relación con la disponibilidad de científicos e ingenieros (v156).

- H14: hay diferencia significativa en los países que conforman la Alianza del Pacífico y el resto de los países de Latinoamérica en relación con las patentes aplicadas (v157).

De acuerdo con las pruebas de hipótesis realizadas, la única variable que mostró una diferencia significativa entre ambos bloques de países fue la aplicación de patentes con un nivel de significancia de 0,048 (tabla 5).

Los valores de las medias en cuanto a las patentes aplicadas para los diferentes grupos de países fueron: Alianza del Pacífico $\mu=2,51$, Latinoamérica sin Alianza del Pacífico $\mu=0,796$, y el promedio para Latinoamérica es $\mu=1,12$ (tabla 6).

En razón de lo anterior, se confirma únicamente la hipótesis H14. El resto de las variables no mostraron diferencias significativas, es decir, son igualmente innovadores los países de Latinoamérica, excepto en las patentes aplicadas. A partir de lo anterior se puede inferir que el bloque de países de la Alianza del Pacífico
Tabla 6

Valores medios de ambos grupos de países en relación a las patentes

\begin{tabular}{lrl}
\hline Descriptivo & $\mathrm{n}$ & Media \\
\hline PCT aplicación de patentes por millón de habitantes & & \\
Latinoamérica sin Alianza del Pacifico & 17 & 0,796161462 \\
Alianza del Pacífico & 4 & 2,510782658 \\
Total & 21 & 1,122755975 \\
\hline
\end{tabular}

Fuente: elaboración propia.

presenta una ventaja competitiva en innovación por su capacidad de

desarrollar patentes (tablas 5 y 6). Dentro de la Alianza, Chile presenta una destacada contribución con una calificación 6,7, en donde la máxima calificación es 7. Indudablemente Chile puede ser un aliado estratégico en la Alianza del Pacífico para transferir modelos de innovación científico-tecnológica reflejados en patentes y que puedan ser capitalizados por el resto de países que conforman la Alianza del Pacífico: Colombia, México y Perú.

\section{Conclusiones}

Los distintos retos que actualmente presentan los países de la Alianza del Pacífico en cuanto a los ámbitos sociales, políticos y económicos han dado cabida a un contexto de limitado espíritu innovador. A tales retos se suma una baja calidad educativa, la falta de relación entre universidades e industria y una casi nula inversión en proyectos de investigación, lo que ha generado un espacio limitado para capitalizar la inventiva y las propuestas de cambio.

Por ende, es necesario que los países miembros de la Alianza del Pacífico se enfoquen a instar a las instituciones de educación superior, a los organismos responsables de la investigación y a los gobiernos y empresas de la región a que promuevan la generación de valor compartido dentro de los procesos de innovación científica tecnológica y la transferencia de conocimiento. Lo anterior, apoyado en un enfoque de competitividad que incluye la cooperación de actores nacionales e internacionales, tal como lo modelan Moon et al. (1995), lo proponen Villarreal y Villarreal (2002) y lo estudian Peña-Vinces y Urbano (2014) en 2 países de Latinoamérica (Perú y Chile).

Algunos de los diferentes organismos que miden la competitividad de las naciones, como el WEF y el IMD, consideran que la innovación es un factor determinante para el desarrollo de las economías nacionales. La innovación es un elemento en el que se requiere hacer claras inversiones por parte de los países en potencial desarrollo, como los que integran la Alianza del Pacífico.

Dentro de la innovación para la competitividad, se ha determinado la relevancia que tienen algunas variables, a diferencia de otras, que parecen tener una relación indirecta o poco significativa. Por ello es que se puede concluir que factores como la capacidad de innovar, la calidad de las instituciones de investigación científica, la adquisición de tecnología avanzada por parte de los gobiernos y la disponibilidad de científicos e ingenieros son aspectos en los que los Estados de la Alianza del Pacífico requieren invertir, pues son los elementos que mayor impacto llegan a tener en el mejoramiento de la competitividad de los países. Esto mismo no se da en el caso de la inversión empresarial y la colaboración universidad-industria, los 
cuales, aunque sí llegan a tener cierta relevancia, esta es indirecta $\mathrm{y}$, por ende, menos significativa.

Algo interesante que surge de la investigación fue la poca influencia que llega a tener la generación de patentes en la región en contraste con los demás elementos. Lo anterior no significa que la generación de patentes no sea un factor relevante para la innovación, sino más bien que la participación que tiene Latinoamérica en este tema es tan mínima, que su realización por parte de los países no consigue mostrar un impacto importante. Sin embargo, este factor resultó ser la única variable en la que se pueden encontrar diferencias entre los miembros de la Alianza del Pacífico y el resto de los países Latinoamericanos, ya que en los demás factores no se mostró una verdadera divergencia en los datos, y se puede decir que resultan igualmente innovadores. A partir de lo anterior se puede inferir, así como lo señala el IMD, que el bloque de países de la Alianza del Pacífico presenta una ventaja competitiva en innovación por su capacidad de desarrollar patentes, aspecto que puede suponer una diferencia significativa, considerando la precariedad de este factor en el resto de la región.

De esta forma, y con base en el presente estudio, se puede enfatizar que el desarrollo científico y tecnológico está claramente vinculado a la innovación para la competitividad y que puede resultar en una estrategia de cooperación interesante entre los países miembros de la Alianza del Pacífico. La innovación ciertamente es uno de los elementos fundamentales para mejorar la competitividad de los países para pasar de economías emergentes a economías basadas en el conocimiento y la generación de valor compartido.

Si consigue impulsarse la generación de patentes, fácilmente podría constituirse como una ventaja competitiva entre los países miembros de la Alianza del Pacífico. Finalmente, se enfatiza en la relevancia que hay de la innovación en este grupo de países y la particular influencia que tiene Chile, país que sin duda alguna puede potenciar el impulso innovador en el gremio. Para ello es muy importante fortalecer las alianzas de colaboración, de tal forma que en la escena global este grupo de países que forman parte de la Alianza del Pacífico resulte ser más potente.

Considerando los anteriores hallazgos es pertinente mencionar que la Alianza del Pacífico ha contemplado dentro de sus proyectos de trabajo la movilidad estudiantil y académica en los países miembros de la Alianza. Este proyecto ya está en su fase de ejecución, y su objetivo es "Contribuir a la formación de capital humano de alto nivel, a través del intercambio académico de estudiantes de pregrado y doctorado, docentes universitarios e investigadores en universidades de los cuatro países» (Alianza del Pacífico, 2016, p. 1). El programa ha beneficiado estudiantes, docentes e investigadores. Sin duda alguna este tipo de estrategias será benéfico para potenciar la innovación. La movilidad de investigadores en estas áreas potenciará la transferencia de conocimiento y generación de patentes en este bloque de países, lo cual posibilitará el tránsito hacia una economía basada en el conocimiento entre los países miembros de la región.

A manera general se concluye que la innovación resulta ser un factor clave para incrementar la competitividad de la Alianza del Pacífico en la arena global. Es necesario que en los próximos años se considere la adopción de un verdadero compromiso de las políticas públicas de estas naciones, que influyan en un sentido de cooperación bajo el nuevo paradigma de competitividad sistémica. Particularmente se precisa una apuesta de recursos humanos de alto nivel y una colaboración palpable entre todos los actores que intervienen en el desarrollo científico-tecnológico de las naciones que constituyen la Alianza.

\section{Conflicto de intereses}

Los autores declaran no tener ningún conflicto de intereses.

\section{Bibliografía}

Alianza del Pacífico. (2016). Temas de Trabajo [consultado 12 Jun 2016]. Disponible en: https://alianzapacifico.net/temas-de-trabajo/

Amorós, J. E., Basco, R. y Romaní, G. (2014). Determinants of early internationalization of new firms: The case of Chile. International Entrepreneurship, 12(1), 283-307.

Amorós, J. E., Felzensztein, C. y Etchebarne, M. (2012). Emprendimiento internacional en Latinoamérica: desafíos para el desarrollo. Esic Market Economics and Business Journal, 43(3), 513-529.

Arredondo, F., de la Garza, J. y Villa, L. (2015). Colaboración organizacional desde la perspectiva del empleado: caso de las maquiladoras del noreste de México. Revista Venezolana de Gerencia, 20(72), 698-716.

Bustos, O. (2015). La innovación basada en ciencia como pilar de desarrollo. El Mercurio, Ediciones Especiales (24 de septiembre de 2015) [consultado 12 Jun 2016]. Disponible en: http://www.edicionesespeciales.elmercurio. com/destacadas/detalle/index.asp?idnoticia=030201509242022

Cámara de Diputados y Diseña México (29 de Octubre de 2008). Memorias del Foro: Por una Política de Diseño para México. México.

Castaños, H. (1994). Universidad e innovación tecnológica. Perfiles Educativos, (65.) [consultado 12 Jun 2016]. Disponible en: http://www.redalyc.org/ html/132/13206505/index.html

Castro-González, S., Peña-Vinces, J. y Guillen, J. (2016). The competitiveness of LatinAmerican economies: Consolidation of the Double-Diamond theory. Economic System, 40 http://dx.doi.org/10.1016/j.ecosys.2015.10.003. En prensa

Charreau, E. (2001). Ciencia e innovación. Interciencia, 26(7), 269

CII. (2011). Programa Nacional de Innovación. México: Presidencia de la Republica.

CIRIEC. (2008). Innovación y economía social. Revista de Economía Pública, Social y Cooperativa, 60, 5 .

De Arteche, M., Santucci, M. y Welsh, S. (2013). Redes y clusters para la innovación y la transferencia de conocimiento. Impacto en el crecimiento regional en Argentina. Estudios Gerenciales, 29(127), 127-138.

Deusto. (2015). Centro de Innovación Social [consultado 12 Jun 2016]. Disponible en: http://www.socialesyhumanas.deusto.es/cs/Satellite/socialesyhumanas/es/ que-es-deusto-innovacion-social/presentacion-7

ESADE. (2015). Instituto de Innovación Social [consultado 12 Jun 2016]. Disponible en: http://www.esade.edu/research-webs/esp/socialinnovation

Ferrari, B. (2012). Innovación Tecnologica: Pilar para el desarrollo [consultado 12 Jun 2016]. Disponible en: http://www.2006-2012.economia.gob.mx/ comunidad-negocios/notas-relevantes/7131-innovacion-tecnologica-pilardesarrollo

Freeman, C. (1993). El reto de la innovación: la experiencia de Japón. Caracas: Galac.

García-Osorio, O., Quintero-Quintero, J. y Arias-Pérez, J. (2014). Capacidades de innovación, desempeño innovador y desempeño organizacional en empresas del sector de servicios. Cuadernos de Administración, 27(49), 87-108.

García-Pérez de Lema, D., Barona-Zuluaga, B. y Madrid-Guijarro, A. (2013). Financiación de la innovación en las Mipyme Iberoamenricanas. Estudios Gerenciales, 29(126), 12-16.

Geroski, P. (1989). Entry, innovation and productivity growth. Review of Economics and Statistics, 71(4), 572-578.

González, T. E. y Martín, M. A. (2013). La innovación en entornos económicos poco favorables: el sector autopartes mexicano. Estudios Gerenciales, 29(127), 167-176.

IMD. (2015). IMD World Competitiveness Ranking [consultado 12 Jun 2016]. Disponible en: http://www.imd.org/news/IMD-releases-its-2015World-Competitiveness-Ranking.cfm

León, J. L. y Ramírez, J. J. (2014). La Alianza del Pacífico. Alcances, competitividad e implicaciones para América Latina [consultado 12 Jun 2016]. Disponible en: http://library.fes.de/pdf-files/bueros/mexiko/11062.pdf

Montoya, O. (2004). Schumpeter, innovación y deterministmo tecnológico. Scientia et Technica, 2(25), 209-213.

Monzón, J. (2010). Entorno para la Innovación. Almeria: Fundación Cajamar.

Moon, H., Rugman, A. y Verbeke, A. (1995). The generalized doble diamond approach to international competitiveness. En A. Rugman (Ed.), Research in Global Strategic Management: A Research Annual (pp. 97-114). Bradford: Emerald Group Published Limited.

Naranjo-Valencia, J. C. y Calderón-Hernández, G. (2015). Construyendo una cultura de innovación. Una propuesta de transformación cultural. Estudios Gerenciales, 31(135), 223-236.

Peña-Vinces, J. (2008). La competitividad internacional en el mundo actual. Lima: Facultad de Ciencias Administrativas. Universidad Nacional Mayor de San Marcos.

Peña-Vinces, J. y Urbano, D. (2014). The influence of domestic Economic Agents on the International Competitiveness of Latin American Firms: Evidence from Peruvian Small and Medium Multinational Enterprises. Emerging Markets Finance and Trade, 50(6), 43-63.

Rivera Godoy, J. A. (2015). Analisis de la relación de la innovación empresarial con la financiación en Colombia. Cuadernos de Administración, 50(28), 11-37.

Sanchez, Y., García, F. y Mendoza, J. E. (2015). La capacidad de innovación y su relación con el emprendimiento en las regiones de México. Estudios Gerenciales, 31(136), 243-252.

Villarreal, R. y Villarreal, R. (2002). México competitivo al 2020. México: Plaza y Valdés.

WEF. (2014). The Global Competitiveness Report 2014-2015. Geneva: World Economic Forum. 